\title{
Balance of a Multijoint Biomechanical System in Natural and Artificial Environments: A Simulation Model
}

\author{
Mikhail P. Shestakov \\ Research Institute of Sport Problems, Russian State University of Physical Education, Moscow, Russia
}

\begin{abstract}
The paper is devoted to a neurobionic simulation model for controlling balance in a biomechanical pendulum. The model is realized by a complex of fuzzy regulators and an artificial neural network. Fuzzy regulators are used for simulating the physiological characteristics of the motor system and the functions of the sensory systems. The second level of control is the central integrator. It is realized as an artificial neural network (ANN), which simulates a real process of analysis and synthesis of afferent signals, formation of the model of action, etc.

Equilibrium control in a multijoint biomechanical object is a specific example of a self-developing multilevel system of movement control. In the course of elaboration of the model and further examination of its behavior we have received model results which revealed correspondence with the results demonstrated by real subjects in stabilographic tests performed after long-term space flights.

We concluded that the model permits us to simulate the peculiarities of human movement control and can be used for creating individual plans of recovery and rehabilitation of patients after long-term motionless or learning movement control in unknown environments. J Physiol Anthropol 26(3): 419-423, 2007 http://www.jstage.jst.go.jp/browse/jpa2
\end{abstract}

[DOI: $10.2114 /$ jpa2.26.419]

Keywords: neuro-fuzzy complex, equilibrium, biomechanical multijoint system

\section{Introduction}

The sensory-motor systems of humans have evolved phylogenetically and ontogenetically to optimize body movements and posture control in the terrestrial gravitational field. The central nervous system (CNS) has developed neurosensory systems which monitor and process sensory inputs from visual, vestibular, somatosensory, and proprioceptive receptors to assess the biomechanical state of the body (spatial orientation), and neuro-motor systems which create, select, and employ motor command strategies and synergies to adjust the biomechanical state towards the desired equilibrium point. Adaptation to microgravity alters neuro-sensory systems by eliminating, reinterpreting, or modifying the weighting of sensory information used to assess spatial orientation in response to the sudden loss of tonic gravitational otolith stimulation (Paloski et al., 1994). Adaptation to microgravity also alters neuro-motor systems by modifying the repertoire of motor command strategies and synergies used for movement control in response to a sudden redistribution of forces along the body, reductions in the biomechanical support reactions, and alteration of relationships between motor command and body movement (Kozlovskaya et al., 1981). These inflight sensory-motor adaptations disrupt postflight postural equilibrium control.

The most difficult task in movement control is to maintain equilibrium of a multijoint system in conditions of gravity and under the influence of various disturbances. In this aspect the system controlling the motor apparatus may be considered as a multilevel control system consisting of several systems controlling separate joints (separate links) (Bernshtein, 1967). When modeling such a system, one should take into account not only the mechanical tasks to be completed, but also the biological peculiarities of the objects primarily based on physiological laws (Shestakov, 2002). Very often these physiological laws are drawn from statistical data, and sometimes their description is not complete. So nowadays, the use of neuro-fuzzy complexes seems to be indispensable for modeling and further examining the laws of information control processes which underlie the movement control of complex biomechanical systems (Shestakov et al., 2004).

The objective of this work was to create a simulation model controlling the equilibrium of a biomechanical multijoint system on the base of neuro-fuzzy complexes and to determine the role of central adaptive mechanisms in reorganizing postural equilibrium control in humans after space flights.

\section{Methods}

For our studies, we have chosen a 3-link system of hinged pivots of equal weight and certain linear dimensions. The 
weight of hinges and friction forces in the system are ignored. The system can move in a single plane only and is maintained in the upright position by elastic ties, two ties for each pivot (Fig. 1). To disturb the equilibrium of the system, we simulated a push into the top pivot.

The simulation model was realized by means of a complex of fuzzy regulators (FRs) and an artificial neural network (ANN) (Shestakov et al., 2004).

Physiological characteristics of elastic ties were simulated with the help of FRs in the system WARPSDT1.0. Fuzzy regulators of different types were distinguished by the type of T-norms, rules of the output, defuzzification, etc. (Averkin, 1987). To calculate weight (or a true value of an antecedent) in WARPSDT all values were combined according to the rule of minimum, or a T-norm. To calculate membership functions of consequence, we used the method of maximal points, which scales the membership function of consequence on the base of the weight of an antecedent using the equation:

$A^{\prime}\left(y_{i}\right)=\min \left(A\left(y_{i}\right) * \alpha_{i}\right)$,

where $\alpha_{i}$ is the level of assurance of the antecedent.

A single fuzzy value of the output variable was calculated using the method of "logical maximum" of the results of application of all the rules.

A modified version of FR, used in the model, is integrated with Delphi and takes into account not only current values of the input parameters, but also values received at the previous step. It allows us to achieve a better simulation of dynamic processes.

Besides simulation of the physiological characteristics of the motor system, FRs are used to simulate sensory systems operation in a biological object. Functions of visual and vestibular entries are simulated in a simplified way by means of corresponding FRs connected to the artificial neural network. Their signals are considered as sources of noise of different magnitude during network "learning".

The second level of equilibrium control in a multijoint system is the central integrator realized as an ANN on the base of the package Brain Maker (California Scientist Software). ANN simulates the real process of analysis and synthesis of afferent signals, develops the model of action, chooses the motor program, triggers motor commands, compares the program being executed with the model of action, generates an error signal, and corrects programs and commands.

$\mathrm{P}$-disturbing force; $\mathrm{S}$ - -sensors (visual and vestibular); M-component model of a muscle (Zatsiorskiy et al., 1997): 1-parallel resilient component, 2-contractile component, a-myosin fibres, b-actin fibres, 3-consecutive resilient component.

Each tie is controlled by a fuzzy regulator, and the whole system is controlled by the ANN. The regulator sends a current value of tension being created by the tie to the neural network (afferentation) and receives the value of tension defined by the network. The final value of the tie tension is determined on the base of the external and internal orientation of the system, its mechanical features (neural network function), and the potential of a tie at any moment (FR function).

\section{Physiological justification of the model controlling the elastic ties}

Approaches used in our research determine 4 principal structures that compose the model-receptors, effectors, central integrator, and memory.

To develop principal mathematical models of muscular work three functions are often used. They are described as a Hilltype model (Heckman and Sandercock, 1996): strength of muscular contraction-frequency of muscular stimulation; strength of muscular contraction-length of the muscle; strength of muscular contraction-velocity of muscular contraction. We have added a fourth function to our model: number of recruited muscular fibers (MF) - muscular tension. The following ratio is observed: slow $\mathrm{MF}>$ intermediate $\mathrm{MF}>$ fast $\mathrm{MF}$ that is often referred to as "principle of dimension" by Henneman.

To verify the correctness of the model, the results of simulation were compared to experimental data registered in the course of the examination of cosmonauts before and after their long-term stay in conditions of microgravity during a Mir-station Mission. The informed consent of all the subjects was obtained prior to the examination.

The stabilographic and electromyographic characteristics of correctional postural responses to disturbances causing the loss of equilibrium were studied with the use of the American postural platform system "Equitest" (NeuruCom, International, Clackamas, OR).

After a long-duration space flight, 9 subjects displayed a disrupted posture control if compared to their preflight performance. Two types of postural disturbance were used. The first one consisted of an unexpected forward displacement $(5.70 \mathrm{~cm}$ in $400 \mathrm{~ms})$ of the support surface (force plate) in the horizontal plane. The second one consisted of graded pushes applied manually (with help of a special tensometric device) to a rigid plate $(10 \times 15 \mathrm{~cm})$ fixed on the chest of the subject. All tests were executed with the subject standing upright on the stabilographic platform force plate with eyes closed. The following indices were registered: native EMG of soleus, tibialis, quadriceps, hamstring, paraspinal and abdominal muscles; force of the disturbance of vertical stance and value of the displacement of the support surface; and data from the force support plates supplied with four sensors registering the force applied by the front and the rear parts of each foot to the support surface of the platform, as well as from two goniometers on hip and shoulder joints.

\section{Results and Discussion}

The main physiological basis of elastic ties operation is the hypothesis of A. G. Feldman (Feldman, 1986), who supposed that an equilibrium point results from interaction of antagonist 
Table 1 Motion strategy changes following long-duration space flight (\% Subjects)

\begin{tabular}{|c|c|c|c|c|}
\hline $\begin{array}{l}\text { Day after flight of } \\
\text { data collection }\end{array}$ & Type of disturbance & $\begin{array}{c}\text { III strategy "Active" } \\
\text { hip sway }\end{array}$ & $\begin{array}{c}\text { II strategy "Passive" } \\
\text { hip sway }\end{array}$ & I strategy Ankle sway \\
\hline \multirow[t]{2}{*}{ Preflight } & Push & 100 & 0 & 0 \\
\hline & Translation & 100 & 0 & 0 \\
\hline \multirow[t]{2}{*}{$\mathrm{R}+0-2$} & Push & 10 & 20 & 70 \\
\hline & Translation & 80 & 0 & 20 \\
\hline \multirow[t]{2}{*}{$R+3-6$} & Push & 40 & 40 & 20 \\
\hline & Translation & 100 & 0 & 0 \\
\hline \multirow[t]{2}{*}{$R+8-10,15$} & Push & 60 & 0 & 40 \\
\hline & Translation & 80 & 0 & 20 \\
\hline
\end{tabular}

muscles in a voluntary movement. The main coordination is considered to be a result of the inherent stretch reflex, and the programming activity of brain in controlling various voluntary movements is thought to be aimed at time and space coordination of the coefficient of reinforcement in the scheme of the realization of this reflex. So reaching the equilibrium point of the stretched muscles provides a necessary movement. The problem is that it is difficult to determine a precise value of dynamic rigidity of a tie at a certain moment of time. Thus, dynamic rigidity of the tie appears to be a fuzzy parameter.

It is impossible to control the whole multijoint system by elements controlling separate ties. To solve this task we need a hierarchical multilevel control system. In our model, separate links are controlled at the lower level of the system. At this level, the principal goal is to realize the law of the equilibrium point (Feldman, 1986), which may be considered as a specific case of maintaining balance of the whole system in the absence of any disturbance. If we set a task to restore a near-balanced state of a multijoint system after a disturbance, we need another control system with different functions. This statement is consistent with the theory of meta-system transitions (Turchin, 1987): a transition from lower to upper levels in the system hierarchy is proceeded by integrating several subsystems of the lower level and forming an integral system at a new level. It does not contradict the theory of functional systems (Anokhin, 1969), assuming that a dynamic integration of the multiple components of living substances leads to positive adaptive results, including execution of voluntary motor actions (Selujanov, 1997).

To simulate the second level of control (central systems from the physiological point of view) we have used ANN.

ANN is a multilayer recurrent network, which is taught to identify interrelations between pairs of elastic ties in each link. Learning is realized by the method of reverse error diffusion. Due to its recurrent character, the network can estimate the results of its work independently and choose the necessary regimen in response to different disturbances.

Depending on the level of training or the strength of disturbance, the equilibrium of the multijoint system is controlled in the primary, program, or approximating regimen of control.

The primary regimen of control consists in a simultaneous growth of forces in all ties of the multijoint system that increases the total rigidity of the system as a whole.

The program regimen of control is developed by the system itself during learning. It can be executed only by a completely learned neural network. In this regimen forces in the ties of each link increase and decrease simultaneously in an alternating mode that enhances the total stability of the system. Estimation of energy expenditures in both regimens demonstrated a considerable advantage in the program regimen of control. The approximating regimen is an intermediate one and characterizes the degree of readiness of the system resulting from learning.

To estimate the adequacy of the model, we verified it using data of the pre- and postflight examination of Russian cosmonauts. The comparative analysis of the examination results and simulation data proved the similarity of strategies of postural equilibrium control in natural and artificial environments.

We found that after a space flight the cosmonauts used three primary motor coordination strategies in response to pushes and translations (Table 1). The strategies after flight were defined by the patterns of segmental body movement responses, and were identified by the first segment to move. The first strategy (I) is the "inverted pendulum" or "ankle sway" strategy, since the only axis of body rotation passed through the ankle joints. This strategy was defined by Kozlovskaya as the "column effect" (Kozlovskaja et al. 1981). The second strategy (II) was a "hip sway" strategy characterized by rotations at the hips and ankles with very little motion of the shoulders. The third strategy (III) was characterized by large extension of the torso (primarily by hip rotation) with little motion of the hips and ankles in space.

Table 1 displays changes in motor coordination strategies after a long-duration space flight (approx. 200 days) (Kozlovskaja et al., 1997). Note that almost all subjects changed the strategy early after flight (at the $\mathrm{R}+0-2$ data collections). There were far more strategy changes in response to pushes than to translations. In the push protocol, the vestibular system receives substantial stimulation; therefore, the vestibular inputs may influence the strategy changes. As the motor system of cosmonauts recovered, the strategies altered in the following order: I-II-III. 
Table 2 Results of "learning" in the model of the biomechanical 3-link system

\begin{tabular}{|c|c|c|c|c|c|}
\hline & \multicolumn{5}{|c|}{ Number of iterations of learning cycles } \\
\hline & 500 & 688 & 1000 & 1500 & 2371 \\
\hline Number of degrees of freedom of the system & 1 & 2 & 2 & 2 & 3 \\
\hline $\begin{array}{l}\text { Total activity of the lower link elastic tie, when the } \\
\text { system returns to the initial position, conventional unit }\end{array}$ & $25,1 \pm 8,4$ & $21,3 \pm 4,3$ & $13 \pm 3,3$ & $12 \pm 2,4$ & $8 \pm 1,8$ \\
\hline Disturbing force, which makes the system "fall", \% & 100 & 123 & 144 & 165 & 215 \\
\hline Maximal safe tilt angle of the system after the disturbance, ${ }^{\circ}$ & $3,0 \pm 0,11$ & $7,4 \pm 1,3$ & $8,0 \pm 0,9$ & $11,3 \pm 0,15$ & $17,7 \pm 0,2$ \\
\hline
\end{tabular}

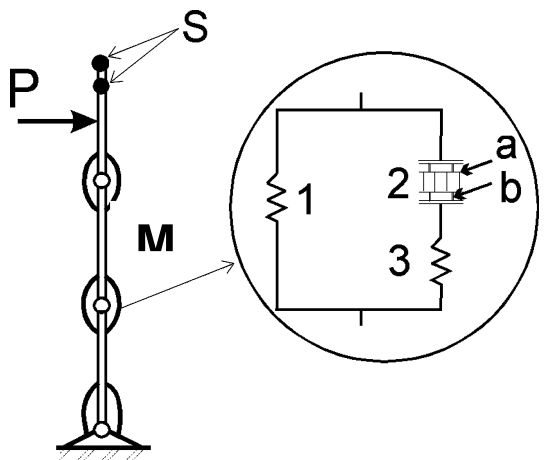

Fig. 1 Scheme of the model of a biomechanical 3-link system.

Simulation results (Table 2) show that in the course of "learning" the model demonstrates the order of alteration of movement control strategies revealed in real experiments. The number of degrees of freedom in the model corresponds to real strategies of postural equilibrium control (Table 1). A system with one degree of freedom (movement in the lower joint, Figure 1) corresponds to the "Ankle sway" strategy (I); movement in a system with 2 degrees of freedom corresponds to the "Passive" hip sway" strategy (II); movement in the three joints of the model corresponds to the "Active" hip sway" strategy (III). On one hand, it is accounted for the optimization of energy expenditures, and on the other hand, it can be explained by a decrease of the control parameters due to the decrease of the number of ties in joint control moments.

\section{Conclusions}

This work is the first one that uses hierarchical neuro-fuzzy simulation models based on parametrical logic.

Fuzzy regulators are used for simulation of the physiological characteristics of the motor system and the functions of the sensory systems. The second level of control is the central integrator realized as an artificial neural network (ANN), which simulates a real process of analysis and synthesis of afferent signals, formation of the model of action, etc.

Equilibrium control in a multijoint biomechanical object is a specific example of a self-developing multilevel system of movement control. During elaboration of the model and further examination of its behavior we have received model results which corresponded to the results of real stabilographic tests.
Our natural experimental results showed that coordinated segmental responses to postural perturbations were altered in nearly all subjects after space flight. We concluded from this that long-duration exposure to microgravity caused substantial changes in central motor programming.

The model allows us to simulate the peculiarities of human movement control involving an interrelation of periphery and center, motor and sensory systems, as well as specificity of development and alteration of motor programs of voluntary movements. The model can help to work out individual plans for the recovery and rehabilitation of patients after long-term motionlessness.

\section{References}

Averkin AN (1987) The fuzzy modelling relation in AI systems. In Ivan P ed. AI and Information-Control Systems of Robots. North-Holland, 165-174

Anokhin PK (1969) Cybernetics and the integrative activity of the brain. In Cole M, Malzmann L eds. A handbook of contemporary Soviet psychology. Basic Books, New York, $830-856$

Bernshtein NA (1967) The coordination and regulation of movements. Pergamon press, Oxford, 1-196

Feldman AG (1986) Once more on the equilibrium-point hypothesis (lambda model) for motor control. J Motor Behav 18: 17-54

Heckman CJ, Sandercock TG (1996) From Motor Unit to Whole Muscle Properties During Locomotor Movements. Exer and Sport Sci Rev 24: 109-133

Kozlovskaja IB, Kreidich AN, Rakham OV (1981) Mechanisms of the effects of weightlessness on the motor system of man. Physiogist (Suppl 24): 59-64

Kozlovskaja I, Shestakov M, Paloski W, Reschke M (1997) Postural disturbances after space flight. J Neurol Sci 150: 338

Paloski WH, Bloomberg JJ, Reshke, Harm DL (1994) Spaceflight-induced changes in posture and locomotion. J Biomec 27: 812

Shestakov M (2002) Biocyberagogics — artificial intelligence in Sport Science of XXI century. 7th Annual Congress European College of Sport Science, Greece, Athens

Shestakov M, Averkin A (2004) Holonic Multi-agent System for Simulation of Locomotor Movements of a Multijoint 
Biomechanical System. Reports of 4-th European Symposium on Intelligent Technologies, Hybrid Systems and their implementation on Smart Adaptive System, Aachen, German, 34-41

Selujanov VN, Shestakov MP (1997) Biomechanism and neuron network as the base of the theory of technical training in sports. Abst of Inter Symp "Brain and movement”. St. Petersburg, Moscow, 167-168

Turchin VF (1987) A constructive interpretation of the full set theory. J Symbolic Logic 52

Zatsiorskiy VM (1997) Kinematics of Human Motion. Human
Kinetics, Champaign, IL, 1-486

This article was presented at the 8th International Congress of Physiological Anthropology, 2006 (ICPA 2006), in Kamakura, Japan.

Received: September 30, 2006

Accepted: March 28, 2007

Correspondence to: Mikhail P. Shestakov, Research Institute of Sport Problems, Russian State University of Physical Education, Moscow, Russia

e-mail: shtv@infosport.ru 